\title{
3D Facial Expression Synthesis: A Survey
}

\author{
Samuel Agianpuye and Jacey-Lynn Minoi \\ Faculty of Computer Science and Information Technology \\ Universiti Malaysia Sarawak \\ Kuching, Malaysia
}

\begin{abstract}
Facial expression synthesis is a process of generating new face shapes from a given face and still retain the distinct facial characteristics of the initial face. The generated facial expressions can be used to improve the performance of existing face identification systems, or to enhance human recognition. Earlier work on synthesizing face shapes used 2D face images. Only recently, the work moved to using 3D face shapes given the availability and improvement in 3D scanner technologies. The advantage of $3 \mathrm{D}$ faces over $2 \mathrm{D}$ image data is that 3D face holds more geometric shape data and is invariant to poses and illumination. This paper aims to give an overview of the methods used for 3D facial expression synthesis. We present an overview of 3D face expression synthesis, its applications and benefits and then we review some of the most resent $3 D$ face expression synthesis approaches.
\end{abstract}

Keywords-Facial expression, 3D faces, synthesis

\section{INTRODUCTION}

Facial expression synthesizing is a growing field of research in computer vision and biometrics. This is because of several advantages it holds, especially in the areas of face recognition and face reconstruction. Facial expression can be described as the movement or position of facial muscles on the skin that could convey the emotional state of an individual. People have a unique ability to understand facial expressions of other individuals. Computers on the other hand do not have this ability. As people become heavily dependent on computers, it will be useful if computers can recognize human facial expressions in order to improve human-computer interaction. This has led to several research interests springing up in the area of computer vision. One of which is synthesizing facial expressions. Synthesizing facial expression simply means artificially creating or replicating human facial expression on an image or 3D face data and in this paper we shall be discussing several approaches to synthesizing realistic human facial expressions, specifically on 3D faces.

Facial expression synthesis has several benefits when applied to certain systems as an add-on module. For example, it can improve the performance of face recognition systems, because a slight expression change can greatly distort recognition accuracy [8][4][7]. Facial recognition is a process of identifying a face by matching it against a library of known faces. An experiment by Chang et al. [7] revealed $90 \%$ recognition rate without any facial expression, but when facial expression was added, the recognition rate dropped significantly to between $25 \%$ to $50 \%$. Synthesizing neutral face expression can be used to solve this problem when implemented in a face recognition environment.
Facial expression synthesis can also be of benefits as an add-on to gender classification algorithms. Existing gender classification methods only deals with neutral faces [14][16]. Neutral facial expression can be synthesized from other kinds of facial expression before gender classification is carried out.

Cartoons or anime production studios can also use facial expression synthesis methods to reduce the amount of frames required to animate a facial expression onto a character's face. Video game developers can also synthesize realistic facial expressions on 3D models and avatars.

Facial expression synthesis can also improve HumanComputer Interaction. For example, in future, front camera sensors on a mobile device can be used to track the facial expression of the user in order to guess his or her emotional state. This information can help the device carry out certain task like telling a joke if it senses the device owner is in a sad mood. It can also be used to improve access control on the mobile device. The mobile user is not required to have a neutral facial expression to $\log$ in. The device can grant access no matter the expression on the user's face. Lastly, facial expression synthesis can serve as add-on to existing aging systems by synthesizing facial expressions used for comparing young and old face data of the individual. These are just a few of the many benefits of facial expression synthesis.

\section{BACKGROUND AND LITERATURE REVIEW}

In order to synthesize facial expression, we need to understand the human facial anatomy and how facial expressions are created. Facial muscle contractions and relaxation in conjunction with the movement of the mandible creates different forms of facial articulations and expressions. As seen in Fig. 1, specific face muscles in conjunction with skull bones, specifically the mandible Fig. 1 (b), are responsible for the generation of various facial expressions on the human face. These sets of muscles deform in specific patterns to create various facial expressions or facial appearance as a whole, like during speech, yawning etc.

Psychologists Paul Ekman and Wallace V. Friesen in 1978 [1] studied the human facial anatomy and created Facial Action Coding System (FACS) framework to explain how facial muscle contractions alter appearance in relation to the creation of expressions. They used Action Unit (AU) to describe each facial muscle contraction or relaxation. All facial expressions can be represented by AUs and can be further described by intensity, asymmetry and duration. Nearly any anatomically possible facial expression can be manually coded by human using FACS. 